\title{
The real implications of reciprocal space artifacts in PDF data analysis
}

Daniel Olds, Joerg Neuefeind, Thomas Proffen, Katharine Page

Spallation Neutron Source, Oak Ridge National Laboratory

Total scattering and pair-distribution function (PDF) methods allow for detailed study of local atomic order and disorder, including materials for which Rietveld refinements are not traditionally possible (amorphous materials, liquids, glasses, nanoparticles). With the advent of modern neutron time of flight (TOF) instrumentation, total scattering studies are capable of producing PDFs with ranges upwards of 10 $-20 \mathrm{~nm}$, covering the correlated length scales of interest for many materials under study. Despite this, the refinement and subsequent analysis of data is often limited by confounding factors that are not rigorously accounted for in conventional analysis programs. We have explicitly explored the effects of resolution, peak-shape, peak-asymmetry, and inconsistent calibration in neutron TOF data as they relate to real-space PDF analysis through simulation. In addition to the simulated studies, we present three case studies of neutron TOF powder diffraction instruments, where the interplay of these effects can be observed. Based on both the simulated analysis and real-world case studies, we present recommend best practices for analysis of data from modern neutron TOF total scattering instruments when using conventional analysis programs, as well as recommendations for improved analysis methods and future instrumentation design. 